\title{
Combined spinal-epidural anesthesia for cesarean section in a patient with Moyamoya disease -A case report-
}

\author{
Kwang Suk Shim, Eun Ju Kim, Ji Hyang Lee, Sang Gon Lee, Jong Seouk Ban, and Byung Woo Min \\ Department of Anesthesiology and Pain Medicine, Fatima Hospital, Daegu, Korea
}

Moyamoya disease is a rare progressive occlusive disease of the internal carotid arteries. We report a case of combined spinal-epidural anesthesia in a patient with Moyamoya disease presenting for Cesarean section. Hypotension associated with spinal anesthesia for Cesarean section is the most common and serious adverse effect despite the use of uterine displacement and volume preload. We continuously infused phenylephrine and ephedrine to prevent hypotension. The intraoperative hemodynamic state was stable. The patient had no significant postoperative complications. (Korean J Anesthesiol 2010; 59: S150-S153)

Key Words: Combined spinal-epidural anesthesia, Ephedrine, Moyamoya disease, Phenylephrine.

Moyamoya disease is a rare vascular disease with an abnormal vessel figure in the cerebral fundus due to the progressive occlusion in the far external parts of the internal carotid artery at both sides $[1,2]$, sometimes accompanied by an intracranial aneurysm. It is mainly found in women and especially pregnant women might have severe neurologic symptoms during the gestational period due to the increased cardiac output and blood hypercoagulability. The presenting symptom is a transient ischemic attack with neurologic abnormality in children and an intracerebral hemorrhage in adults $[2,3]$.

In the case of Moyamoya disease accompanied by pregnancy, since hyperventilation and an increased blood pressure due to the labor pain can increase the risk of cerebral ischemia and aneurysm rupture $[1,4]$, selective Cesarean section has been known to be appropriate rather than vaginal delivery [1]. There is no agreement in the methods of anesthesia, including general anesthesia, spinal anesthesia, and peridural anesthesia.

Since we have experienced a successful delivery and postoperative pain control for a 27 -year-old primipara by the spinal-peridural anesthesia and the continuous infusion of phenylephrin and ephedrine mixed with physiological saline to prevent hypotension, we herein report the case with a related literature survey.

\section{Case Report}

A 27-year-old primipara at the 38th week of pregnancy visited the neurosurgery department with the complaint of a headache that continued from 2 years ago. She was diagnosed with Moyamoya disease and followed-up in the neurosurgery

Received: April 15, 2010. Revised: April 23, 2010. Accepted: May 9, 2010.

Corresponding author: Eun Ju Kim, M.D., Department of Anesthesiology and Pain Medicine, Fatima Hospital, 302-1, Sinam-dong, Dong-gu, Daegu 701-010, Korea. Tel: 82-53-940-7434, Fax: 82-53-954-7417, E-mail: kej1127@fatima.or.kr

(c) This is an open-access article distributed under the terms of the Creative Commons Attribution Non-Commercial License (http:// creativecommons.org/licenses/by-nc/3.0/), which permits unrestricted non-commercial use, distribution, and reproduction in any medium, provided the original work is properly cited. 
department. No other neurologic symptom was found except the hemorrhage in the left lateral cerebral ventricle by the brain MRI.

Cesarean section was carried out in the 38th week of pregnancy. The patient's consciousness was lucid and the preoperative physical examination results were weight $77.5 \mathrm{~kg}$, height $167 \mathrm{~cm}$, blood pressure 120-140/60-80 $\mathrm{mmHg}$, and heart rate $62-80 / \mathrm{min}$. The blood test results were hemoglobin 12.0 $\mathrm{g} / \mathrm{dl}$, platelet 159,000/ $\mathrm{ll}$, and PT/aPTT 9.8 (INR 0.89)/23.4. The findings from all the other tests, including electrocardiography and chest $\mathrm{X}$-ray imaging, were in the normal range.

For the preanesthetic treatment, an intravenous injection of famotidine $20 \mathrm{mg}$ and an intramuscular injection of glycopyrrolate $0.2 \mathrm{mg}$ were carried out 30 minutes beforethe operation. The heated, lactated Ringer's solution $700 \mathrm{ml}$ was intravenously injected after the patient's arrival in the operating room. The patient's blood pressure was $120 / 80 \mathrm{mmHg}$ and heart rate was $65 / \mathrm{min}$ after arriving in the operating room. Before the peridural anesthesia, a radial artery catheter was inserted to start the continued blood pressure monitoring.

After letting the patient be in the left lateral recumbent position, the epidural space was verified with the loss of resistance method usingan $18 \mathrm{G}$ Tuohy paracentesis needle by the median lumbar puncture in between L4-5 lumbar spines, and the catheter was installed and fixed. After an injection of $1.5 \%$ lidocaine $3 \mathrm{ml}$, the tested amount, containing $1: 200,000$ epinephrine, no increase in the heart rate or spinal anesthesia symptom was found. To make the total volume of phenylephrine and ephedrine $20 \mathrm{ml}$, phenylephrine $0.5 \mathrm{mg}$ and ephedrine $30 \mathrm{mg}$ were mixed with physiological saline and infused at the flow rate of $40 \mathrm{ml} / \mathrm{hr}$.

Thereafter, dural tapping was carried out using a $25 \mathrm{G}$ paracentesis needle by the median lumbar puncture method in between the L5-S1 lumbar spines. After checking the cerebrospinal fluid, the intraspinal injection of $0.5 \%$ bupivacaine $7 \mathrm{mg}$ and fentanyl $20 \mu \mathrm{g}$ followed. After changing the patient's position into the supine position, the right hip was supported with a pillow so that the uterus could be tilted toward the left. The operation started after checking that the sensory deprivation reached $\mathrm{T} 4-5$ by the pinprick test.

The intraoperative vital signs were relatively stable with the blood pressure of 120-150/60-80 mmHg and the heart rate of $65-85 / \mathrm{min}$. A Venti ${ }^{\circledR}$ ask, from which the oxygen fraction of inspired air was $35 \%$, was applied, and a capnometer was installed below the patient's nose to monitor the capnogram. The oxygen saturation measured with a pulse oximeter was $100 \%$. The continued infusion of phenylephrine and ephedrine was stopped 15 minutes after the spinal anesthesia because the blood pressure was kept stable.

A 2,900 g healthy female infant was given birth and the
Apgar score of the newborn child was 9 at 1 minute and 10 at 5 minutes. The patient did not complain of any discomfort and the total duration of the operation was 1 hour. The total amount of the fluid infused during the operation was 2,000 ml and the amount of urine was $1,100 \mathrm{ml}$. For the postoperative desensibilization, the patient-controlled epidural analgesia was performed by mixing morphine $8 \mathrm{mg}$ and fentanyl $500 \mathrm{mcg}$ with $0.125 \%$ ropivacaine $100 \mathrm{ml}$, totally $118 \mathrm{ml}$, and infusing the mixture at the flow rate of $1.5 \mathrm{ml} / \mathrm{hr}$. The vital signs in the recovery room were stable. The Visual Analogue Scale (VAS) was maintained at 0 to 1 during the hospitalization. The patient was discharged on the fifth day after the operation without any specific finding and is now followed up as an outpatient in the neurosurgery department.

\section{Discussion}

Moyamoya disease is a rare disease that brings about stricture and progressive occlusion in internal carotid arteries at both sides. The disease invades the far external parts that are the procephalic, mesencephalic, and cerebral arteries, and eventually the blood supply to the parenchyma becomes dependent on the lateral angiogenesis from the vertebral artery. Such lateral angiogenesis is characterized by a tobacco fume pattern on the angiography $[1,2]$.

Family history implies that genetic factors are involved in the disease to some extent. The frequency of the disease occurrence is relatively high in young women. Various symptoms are found in the disease. Neurologic abnormalities such as transient ischemic attack, paroxysmal hemiplegia, hemiamyosthenia, involuntary movement, and epileptic seizure are found in children. The neurologic abnormalities are more frequently found in children when hyperventilation is caused by crying, coughing, or eating hot food. The abnormalities are due to the decrease of the cerebral blood flow and the higher cerebral oxygen consumption rate than occurs in adults. The cardinal symptoms in adults are headache and disturbance of consciousness due to cerebral ischemia or intracerebral hemorrhage. A cerebral aneurysm also sometimes occurs in the case of adults [2,3].

In the case of Moyamoya disease accompanied by pregnancy, the significant fact is that the probability of neurologic abnormalities exacerbation and an intracerebral hemorrhage is increased. In this case report, an intracerebral hemorrhage was found in the left lateral cerebral ventricle. The hypercoagulable state induced by pregnancy and hypocapnia caused by hyperventilation in the delivery procedure are more likely to bring about cerebral ischemia. In addition to the risk of hyperdynamic circulation due to pregnancy and increased abdominal pressure from labor pain, the risk of an intracerebral hemorrhage due to 
hypertension is increased, as well as the danger of rupture when accompanied by an aneurysm $[4,5]$. Thus, selective Cesarean section has been known to be appropriate rather than vaginal delivery [1].

There is no agreement in the methods of anesthesia. Various cases have been reported in general anesthesia [1,6], spinal anesthesia [7], and peridural anesthesia [8-10]. The objective anesthesia control is to maintain the balance between the cerebral blood flow and the cerebral oxygen consumption rate. Although the protective effect on the ischemia part can be expected since inhalation anesthetics reduce the cerebral metabolic rate in the case of general anesthesia [11], there is risk of pulmonary aspiration during tracheal intubation, intracerebral hemorrhage due to the increased blood pressure, and fetal distress. It is difficult to evaluate the cerebral function with respect to the intraoperative intracerebral hemorrhage or cerebral ischemia. Non-invasive cerebral oxygen saturation monitoring such as INVOS ${ }^{\circledR}$ an help the prediction of intraoperative intracerebral hemorrhage or cerebral ischemia [6], but the sensitivity and specificity of the monitoring method are limited. In the case of spinal anesthesia and peridural anesthesia, the risk of blood pressure increases while fetal distress and pulmonary aspiration decrease. The cerebral function can be evaluated and abnormal neurologic symptoms can be detected by observing the patient's consciousness. Peridural anesthesia has advantages such that the hemodynamic change is gradual in comparison with spinal anesthesia and additional anesthetic injection is possible, but the induction failure rate is higher than that of spinal anesthesia. Spinal anesthesia bears the risk of cerebral ischemia due to the hypotension induced by the sympathetic block.

In this case report, we chose the spinal-peridural joint anesthesia and carried out a continuous infusion of phenylephrin and ephedrine mixed with physiological saline to prevent hypotension [12]. The spinal-peridural joint anesthesia provides several advantages such that blood pressure elevation that may be caused by laryngoscope insertion and endotracheal intubation in general anesthesia can be avoided; the mother's pulmonary aspiration and the fetal distress can be prevented; the cerebral function can be evaluated; and the abnormal neurologic symptoms can be detected by observing the patient's consciousness. When compared to the case of performing either peridural anesthesia or spinal anesthesia alone, the spinal-peridural joint anesthesia provides rapid expression and a stable blocking effect, which are the advantages of spinal anesthesia, as well as control of the blocking level, extension of anesthesia, and easy postoperative pain control, which are the advantages of peridural anesthesia. However, caution should be given to the risk of cerebral ischemia due to hypotension when carrying out the spinal-peridural joint anesthesia. In this case, the fluid volume preload and the uterine displacement were performed to avoid hypotension and the blood pressure was continuously monitored by the radial artery catheter. However, these measures were not enough to prevent hypotension, so phenylephrin and ephedrine mixed with physiological saline were continuously infused. Concerning the hypertensor that is continuously infused to prevent hypotension, Mercier et al. [13] reported that the hypotension frequency in the group to which an ephedrine-phenylephrine mixture was infused was less than that of the group to which only ephedrine was infused. Jung et al. [12] reported that the hypotension prevention and postoperative nausea and vomiting suppression were more effective in the group to which only phenylephrine was infused and the group to which a phenylephrine-ephedrine mixture was infused than in the group to which only ephedrine was infused. Based on these results, phenylephrine $0.5 \mathrm{mg}$ and ephedrine $30 \mathrm{mg}$ were mixed with physiological saline to make the total volume of phenylephrine and ephedrine $20 \mathrm{ml}$. The mixture was infused at the total flow rate of $40 \mathrm{ml} / \mathrm{hr}$ so that the flow rate of phenylephrine and ephedrine could be $16.6 \mu \mathrm{g} / \mathrm{min}$ and $1 \mathrm{mg} / \mathrm{min}$, respectively.

Hypocapnia by hyperventilation should be avoided since it can also cause cerebral ischemia. We installed a capnometer below the patient's nose to monitor the capnogram. Hypocapnia by hyperventilation was avoided and the body temperature was kept at $35.2-36.6^{\circ} \mathrm{C}$ by maintaining the $\mathrm{PaCO}_{2}$ at $30-40 \mathrm{mmHg}$ with periodic arterial blood gas analysis through the radial artery catheter.

Moreover, in order to reduce the risk of complications, such as intracerebral hemorrhage, due to the increased blood pressure induced by the postoperative pain, a patient-controlled epidural analgesia was performed.

In conclusion, we report the case where the selective Cesarean section of a pregnant woman diagnosed with Moyamoya disease was successfully carried out by simultaneously applying spinal-peridural joint anesthesia and continuously infusing phenylephrin and ephedrine mixed with physiological saline to prevent hypotension. In addition, we have given a related literature survey.

\section{References}

1. Miyakawa I, Lee HC, Haruyama Y, Mori N, Mikura T, Kinoshita K. Occlusive disease of the internal carotid arteries with vascular collaterals (Moyamoya disease) in pregnancy. Arch Gynecol 1986; 237: 175-80.

2. Suzuki J, Kodama N. Moyamoya disease - a review. Stroke 1983; 14: 104-9.

3. Aoki N, Mizutani H. Does moyamoya disease cause subarachnoid hemorrhage? Review of 54 cases with intracranial hemorrhage confirmed by computerized tomography. J Neurosurg 1984; 60: 
348-53.

4. Wiebers DO. Ischemic cerebrovascular complications of pregnancy. Arch Neurol 1985; 42: 1106-13.

5. Enomoto H, Goto H. Moyamoya disease presenting as intracerebral hemorrhage during pregnancy - case report and review of the literature. Neurosurgery 1987; 20: 33-5.

6. Mun SH, Kim JH, Woo CH, Kim YH, Kim HS. General Anesthesia for cesarean section in a patient with Moyamoya disease and Sick sinus syndrome. Korean J Anesthesiol 2006; 50: 232-5.

7. Llorente de la Fuente A, Gimenez Garcia MC, Lopez Sanchez F. Regional anaesthesia in moyamoya disease. Br J Anaesth 1997; 78: 478-9.

8. Oh MK, Kim KH, Kim DW. Two cases of epidural anesthesia for patient with Moyamoya disease presenting for elective cesarean section. Korean J Anesthesiol 1999; 36: 558-62.

9. Choi DH, Lee JJ, Ahn HJ. Epidural Anesthesia for cesarean section in a patient with Moyamoya disease. Korean J Anesthesiol 1998; 34:
434-8.

10. Kato R, Terui K, Yokota K, Nakagawa C, Uchida J, Miyao H. Anesthetic management for cesarean section in moyamoya disease: a report of five consecutive cases and a mini-review. Int J Obstet Anesth 2006; 15: 152-8.

11. Smith AL, Wollman H. Cerebral blood flow and metabolism: effects of anesthetic drugs and techniques. Anesthesiology 1972; 36: 378400 .

12. Jung SW, Kim EJ, Min BW, Ban JS, Lee SG, Lee JH. Comparison of maternal and fetal effects of Ephedrine and Phenylephrine infusion during spinal anesthesia for cesarean section. Korean J Anesthesiol 2006; 51: 335-42.

13. Mercier FJ, Riley ET, Frederickson WL, Roger-Christoph S, Benhamou D, Cohen SE. Phenylephrine added to prophylactic ephedrine infusion during spinal anesthesia for elective cesarean section. Anesthesiology 2001; 95: 668-74. 